\title{
Precautions That We Are Observing One Transport System Unobscured by the Contributions of Others
}

\author{
HALVOR N. CHRISTENSEN \\ Department of Biological Chemistry, The University of Michigan \\ Ann Arbor, Michigan 48109
}

\begin{abstract}
Over the past fifteen years the necessity has come to be recognized of differentiating between two or more mechanistically independent contributions to the transport of a given solute into or out of a cell or organelle, if we are to have a clear picture of the operation of any one of the mediating systems. I have been asked to discuss this necessity and the procedures for meeting it. Occasionally it is supposed that the simplest kinetic plots showing linearity or non-linearity of $\mathrm{l} / \mathrm{v}$ as a function of $\mathrm{l} / \mathrm{s}$ ] are sufficient evidence for homogeneity or duality of transport mediation. In actuality, profound heterogeneity can be hidden in a plot that seems to be satisfied by one or two straight lines. Conversely, a bend in such a plot provides inadequate evidence that two distinct agencies are at work. On the contrary, each supposed contribution needs to be shown to proceed either unchanged or changed in a predictable way when the other contributions are prevented. Furthermore, that prevention should best be obtained in more than one way, including not only mutation where that is possible, but also in ways that describe functional differences in the parallel routes.
\end{abstract}

Figure 1 represents an idealized, independent inhibition of each of two transport components, applied first in one sequence and then in the other. In applying this approach to a set of analogous substances, e.g., the neutral amino acids, we seek to discover features of the structures of analogs that bar them from one system but not from the other, to approach the sketched ideal. This approach can be illustrated by three cases:

1. The dichotomy between $\mathrm{Na}^{+}$-dependent System $A$ and $\mathrm{Na}$-independent System $L$ in the Ehrlich cell, where the feature of $\mathrm{N}$-methylation has served to restrict transport to the first, and the feature of 3-dimensional sidechain bulk, to the second. Substitution of choline for $\mathrm{Na}^{+}$in the environment and $\mathrm{pH}$ modification can supplement this approach.
2. The dichotomy between two $\mathrm{Na}^{+}$dependent systems, $A$ and ASC, by the intolerance of the latter to N-methylation or $\mathrm{Li}^{+}$substitution for $\mathrm{Na}^{+}$. This study permitted localization of the $\mathrm{Na}^{+}$-recognizing subsite in juxtaposition to the amino acid, and contrast of that position with the one taken by cations or cationic groups in other transport systems.

3. The dichotomy between an arginine-specific system and a general system for cationic amino acids in Salmonella typhimurium, as shown recently by Quay and Christensen. Histidine inhibits only the first, homoserine or trans-4-hydroxyproline only the latter. The action of the last-mentioned analog (which does not serve as a substrate) permitted Quay and Christensen to show what feature of the guanidinium group on its sidechain permits arginine to be specifically recognized.

In the Ehrlich cell the molecular transport of glutamic acid and its homologs is largely restricted to a handicapped use of neutral Systems $A$ and $L$, a true anionic system being absent from this cell. System $A$ loses activity by its own protonation, so that acidification to protonate the sidechain of glutamic does no good. In contrast, System $L$ becomes reactive with these anionic amino acids only as the charge on the sidechain can be dispelled. A normal preference is shown for the $L$ isomers, except that a reversed stereospecificity is seen for the slower uptake of aspartic acid. This difference resides significantly in $V_{\max }$, not $\mathrm{K}_{\mathrm{m}}$. This finding promises to become highly informative. Interpretation of dicarboxylic amino acid transport in this cell had not succeeded until the two components could be seen separately. Furthermore, the selectivity of System $L$ is so substantially changed on lowering the $\mathrm{pH}$ to 5 or less that establishing it as the mediating agency was not easy. For example, 2-aminoisobutyric acid, ordinarily largely specific to System $A$, becomes a System $L$ substrate, thus adding force to our prior 

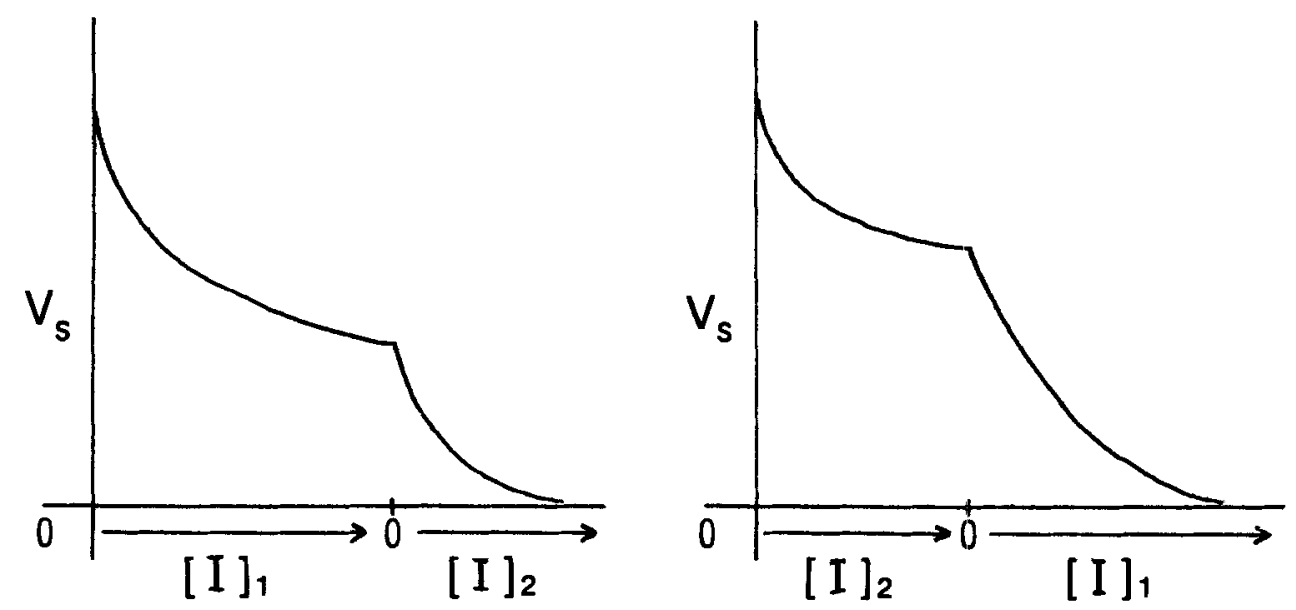

Fig. 1 Idealized diagram of independent inhibition of two transport components.

preference for its $\mathrm{N}$-methyl derivative as the model substrate for System $A$.

A tabular summary (Christensen, '75, table 1) emphasizes that among the ordinary amino acids it is scarcely correct to think of some as System $L$ substrates, others as System $A$ substrates, because all or almost all have affinity for both systems. What is the meaning of this duality? We tend to make our observations under conditions that show each system operating for uptake, although we have been able to show that all the systems studied can produce net exodus as well as entry. Perhaps our most serious error in dealing with the duality or multiplicity of transport systems lies in our bad summations of their contributions to the two fluxes, inward and outward - indeed we invent additional exodus or [shudder!] "retention" systems because we fail to see that we already have all we need. We suppose that a weakly concentrative system can help a strongly concentrative system, whereas the actual relation at the steady state will be that the first of these comes to serve for net exodus, and the stronger system, for net entry. This relation has obvious functional value for the epithelial cells of the intestinal or renal tubular mucosa, in which the steeply uphill, $\mathrm{Na}^{+}$-dependent systems at the brushborder pole of the cell can dominate over the opposed operation of a statistically weaker population of pumps at the contralateral surface. On the surfaces of nonepithelial cells the weaker and stronger pumps may instead be randomly distributed. The superior control of intracellular levels thus achievable may be an important gain arising from an exodus opposed through a reversed passage through a pump. Furthermore, since we are indeed speaking of a reversed operation of the transport process, part of the energy invested in uptake is probably recovered chemiosmotically in exodus. Without the discrimination of the simultaneous contribution of two or more systems to transport, we would exclude ourselves from an understanding of these flux relations and their significance.

I have discussed elsewhere the importance of discriminating distinct components of transport for the interpretation of its biological regulation (Christensen, '73). It is striking how regularly the system subject to regulation of various kinds has proved to correspond to our description of System A. In one instance (Cecchini et al., '76), an inhibition of System $A$ on cell confluency is actually accompanied by stimulation of System $L$, two influences that should cooperate for the regulation of the cytoplasmic amino acid levels.

\section{LITERATURE CITED}

Cecchini, G., M. Lee and D. L. Oxender 1976 Transport of amino acids in intact $3 \mathrm{~T} 3$ and SV3T3 cells: Binding activity for leucine in membrane preparations of Ehrlich ascites tumor cells. J. Supramolecular Structure, 4: 441-447.

Christensen, H. N. 1.973 On the development of a mino acid transport systems. Fed. Proc., 32: 19-28.

1975 Recognition sites for material transport and information transfer. Current Topics in Membrane Transport, 6: 229. 